\title{
SIMULATIONS OF LASER PULSE COUPLING AND TRANSMISSION EFFICIENCY IN PLASMA CHANNELS
}

\author{
R. E. Giacone, D. A. Dimitrov, D. L. Bruhwiler, P. Messmer and J. R. Cary \\ Tech-X Corporation, 5621 Arapahoe Ave., Suite A, Boulder, Colorado 80303 \\ C. G. R. Geddes, E. Esarey and W. Leemans \\ Lawrence Berkeley National Laboratory, 1 Cyclotron Road, Berkeley, California 94720
}

\begin{abstract}
Optical guiding of a laser pulse in the laser wake field accelerator (LWFA) via plasma channels can greatly increase the interaction length and, hence, the maximum energy of trapped electrons [1]. Energy efficient coupling of laser pulses from vacuum into plasma channels is very important for optimal LWFA performance. We present 2D particlein-cell (PIC) simulations of this problem using the VORPAL code [2]. Some of the mechanisms considered are enhanced leakage of laser energy transversely through the channel walls, enhanced refraction due to tunneling ionization of neutral gas on the periphery of the gas jet, ionization of neutral gas by transverse wings of the laser pulse and effect of the pulse being off axis of the channel. Using power spectral diagnostics [3], we are able to differentiate between pump depletion and leakage from the channel. The results from our simulations show that for short $\left(\approx \lambda_{p}\right)$ plasma ramp, very little leakage is seen and pump depletion is roughly 4 times larger than expected from 1D theory [4]. For narrow channel walls and long ramps, leakage increases significantly.
\end{abstract}

\section{INTRODUCTION}

The laser wake field accelerator (LWFA) can excite and sustain very high longitudinal electric fields (greater than $100 \mathrm{GV} / \mathrm{m}$ ) that may allow acceleration of particles to very high energies (greater than $1 \mathrm{TeV}$ ) in extremely short distances (few millimeters). More precisely, ionized plasmas can sustain electron plasma waves (EPW) with longitudinal electric field on the order of the non relativistic wavebreaking field, $E_{0}=c m_{e} \omega_{p} / e$. For a typical electron density of $n_{e}=10^{18} \mathrm{~cm}^{-3}$ the electric field is $E_{0} \approx 100 \mathrm{GV} / \mathrm{m}$ which is approximately three orders of magnitude greater than obtained in conventional RF linacs. A correctly placed trailing electron bunch can be accelerated by the longitudinal electric field and focused by the transverse electric field of the plasma wake. Reaching a $1 \mathrm{GeV}$ LWFA module has emerged as a common goal for the plasma-based accelerator community. In order to reach $1 \mathrm{GeV}$ energies, the laser must be kept focused over many Rayleigh lengths. Because diffraction limits acceleration length to about one Rayleigh length, guiding of a laser beam as it propagates in plasma is necessary. Laser guiding in plasma channels has been proposed as a means to overcome the limitation on the acceleration distance due to pulse diffraction by ex- tending the acceleration length. Energy efficient coupling of laser pulses in plasma channels is of critical importance for optimal laser wake field acceleration. In this paper we have studied these issues by performing 2D PIC simulations using the VORPAL code. We studied the coupling of the laser pulse with the channel by varying the length of the plasma ramp and position of the laser focus inside the density ramp. Oscillations of the laser spot size inside the channel are tracked to determine when there is an optimum matching between the pulse and channel. Using power spectral diagnostics we determined how pump depletion and leakage are affected by inefficient coupling.

The results from our simulations showed that when the laser pulse is focused at the top of the density ramp, matching between the pulse and channel is not efficient and as a result, big amplitude oscillations of the laser spot size occur inside the channel. The power spectral diagnostics show that pump depletion and leakage increase with the amplitude of the spot size oscillations.

\section{ENERGY COUPLING OF LASER PULSES IN A PLASMA CHANNEL}

In this section we present 2D PIC simulations results obtained by using the VORPAL code to study the coupling of the laser pulses into plasma channels. In a typical experimental set up, the entrance of the plasma channel is preceded by a density ramp that can be of many Rayleigh length long. A diffraction limited laser pulse is usually focused at the channel entrance. The results of our simulations showed that there is considerable energy lost when the density ramp is long (more than one Rayleigh length) and the laser is focused at the entrance of the channel due to poor matching between the laser pulse and channel. This mismatch can be eliminated or greatly reduced by focusing the laser pulse before the channel entrance. We begin our investigation by performing 2D PIC simulations varying the length of the plasma density ramp from one to three Rayleigh length. First, the laser pulse was focused at the entrance of the channel. Then, we refocused the pulse to minimize mismatching with the plasma channel. The evolution of the spot size inside the channel is tracked and pump depletion and leakage is accounted for using power spectral diagnostics.

In all simulations, the laser pulse is taken to be linearly polarized, Gaussian in the transverse direction, and with an 
amplitude variation that is longitudinally a half-sine pulse,

$$
\begin{aligned}
E(x, y, t)= & E_{0} \sqrt{\frac{W_{0}}{W(x)}} \exp \left[-\frac{y^{2}}{W(x)^{2}}\right] \\
& \cos \left[k_{0} x-\omega_{0} t+\frac{k_{0} y^{2}}{2 R(x)}-\frac{\phi(x)}{2}\right](1)
\end{aligned}
$$

where $W(x)=W_{0} \sqrt{1+\left(x-x_{f}\right)^{2} / Z_{R}^{2}}$ is the width of the pulse, $W_{0}$ is the width at the location of focus $x_{f}$ and $Z_{R}=k_{0} W_{0}^{2} / 2$ is the Rayleigh length. Of course, the pulse characteristics are actually determined on only the boundary of the simulation, and the dynamics determines the pulse evolution once the pulse is inside the simulation region. The laser pulse used in our simulations was chosen to have wavelength $\lambda_{0}=0.8 \mu \mathrm{m}$, amplitude $a_{0}=0.7$, length $L_{0}=30 \mu \mathrm{m}$ and spot size $r_{0}=6 \mu \mathrm{m}$. The Raleigh length is $Z_{R}=141.0 \mu \mathrm{m}$.

The laser pulse is launched into a $30 \mu \mathrm{m}$ vacuum region, followed by a ( 1, 1.5, 2, 3 Rayleigh length) density ramp with a $(1$ - cosine) shape for the ramp and a peak density $n_{e 0}=1.2386 \times 10^{24} \mathrm{~m}^{-3}$, which corresponds to a plasma wavelength of $30 \mu \mathrm{m}$ and to a plasma frequency of $\omega_{p}=6.297 \times 10^{13} \mathrm{~s}^{-1}$. The density ramp is followed by a preformed fully ionized plasma channel. The channel is modeled by a transverse parabolic density profile, $n(r)=n_{0}+\delta n\left(\frac{r}{r_{0}}\right)^{2}$, where $\delta n=\left(\pi r_{e} r_{s}^{2}\right)^{-1}$ is the critical depth [5, 6], and $r_{e}=e^{2} / m_{e} c^{2}$ is the classical electron radius. The value of $\delta n=1.22 \times 10^{25} \mathrm{~m}^{-3}$ is about 4 times larger than $\delta n_{c}=3.13 \times 10^{24} \mathrm{~m}^{-3}$ which is the theoretical value for guiding in the absence of nonlinearities.

The simulation region varies from $L_{x}=320$ to $500 \mu \mathrm{m}$ in the $x$ direction and from $L_{y}=58$ to $121 \mu \mathrm{m}$ in the $y$ direction, and we used five particles per cell at full density. A moving window was also used in the simulations. Fig. 1 shows the laser spot size as propagates inside the plasma ramp and channel for different ramp lenghts. The stared plot corresponds to the case where the pulse is focused at the top of the density ramp. It can be noticed that even though we chose the pulse parameters to match the channel ones, the big oscillations observed in Fig. 1 by the spot size is an indication of a mismatch between channel and pulse. We also observed that at the focused position the spot size is smaller than the minimum spot size $\left(r_{0}=6 \mu \mathrm{m}\right)$, which is an indication that the pulse is experienced self-focusing instability as propagates in the density ramp. In the next section it will be shown how the mismatch degrades the energy coupling between the pulse and channel. The effects of self-focusing can be offset by focusing the laser pulse before the channel entrance. The diamond line in Fig. 1 shows the spot size corresponding to a focus position about $70.0 \mu \mathrm{m}, 100.0 \mu \mathrm{m}$ and $140.0 \mu \mathrm{m}$ respectively before the channel entrance. We clearly observed the self focusing is compensated and a good matching is obtained. As a result, the spot size is kept fairly constant as the laser pulse propagates inside the channel. We also observed that when the density ramp is longer, the amplitude of the oscillations
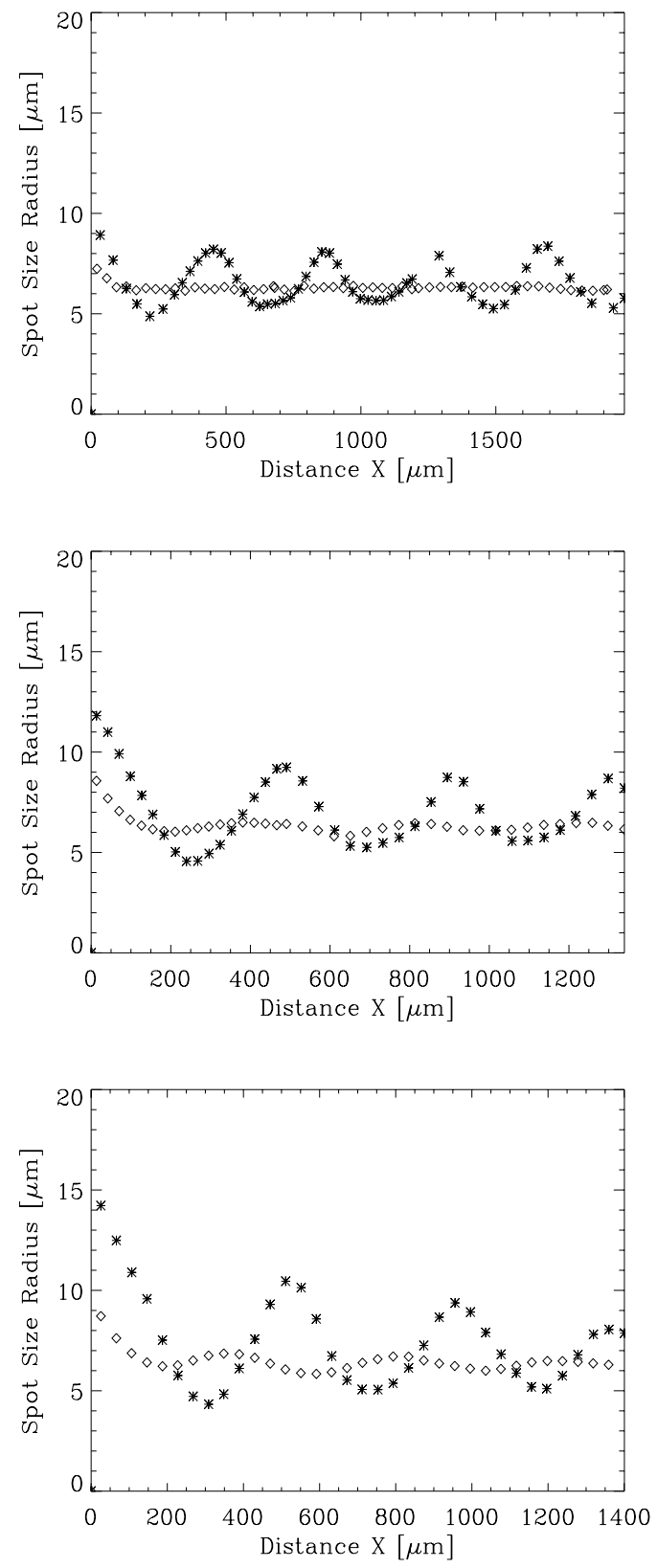

Figure 1: Pulse spot size as a function of propagation distance for the 1, 1.5 and 2 Rayleigh length density ramp case. The star line shows the spot size when the laser is focused at the entrance of the density channel. Diamond line corresponds to placing the focus before the top of density ramp to eliminate spot oscillations.

and self-focusing effects are more pronounced.

\section{DIAGNOSTICS FOR ENERGY LEAKAGE AND PUMP DEPLETION}

A laser pulse propagating inside a plasma will gradually loose part of its energy reducing the efficiency of the LWFA and the maximum energy the electrons can be accelerated. 


\begin{tabular}{|l|l|l|l|c|c|}
\hline \hline Ramp Length & Diagnostic Position & {$[-2 \sigma,+2 \sigma]$} & All Ny & Pump Depl (\%) & Leakage (\%) \\
\hline Vacuum & - & 1.4498239 & 1.4498239 & & \\
$1 Z_{R}(141 \mu \mathrm{m})$ & Top Ramp & 1.4223095 & 1.4495481 & 0.019 & -1.8788 \\
$1 Z_{R}$ & After 7 $Z_{R}(1173 \mu \mathrm{m})$ & 1.3295906 & 1.4398162 & 0.6903 & -7.6027 \\
$1 Z_{R}$ & After 8 $Z_{R}(1313 \mu \mathrm{m})$ & 1.2932571 & 1.4386848 & 0.7683 & -10.0307 \\
$1.5 Z_{R}(211 \mu \mathrm{m})$ & Top Ramp & 1.4376028 & 1.4480038 & 0.1255 & -0.7174 \\
$1.5 Z_{R}$ & After 6.5 $Z_{R}(1120 \mu \mathrm{m})$ & 1.3395672 & 1.4405299 & 0.6410 & -6.9638 \\
$1.5 Z_{R}$ & After 7.6 $Z_{R}(1322 \mu \mathrm{m})$ & 1.2495343 & 1.4368243 & 0.8966 & -12.9181 \\
$2 Z_{R}(282 \mu \mathrm{m})$ & Top Ramp & 1.4358527 & 1.4471254 & 0.1861 & -0.7775 \\
$2 Z_{R}$ & After 6.5 $Z_{R}(1221 \mu \mathrm{m})$ & 1.3212955 & 1.4341269 & 1.0827 & -7.7824 \\
$2 Z_{R}$ & After 8 $Z_{R}(1425 \mu \mathrm{m})$ & 1.1706534 & 1.426558 & 1.6047 & -17.6507 \\
\hline \hline
\end{tabular}

Table 1: Power Spectral Diagnostic for varying ramp lengths and focus at the channel entrance

\begin{tabular}{|l|l|l|l|c|c|}
\hline \hline Ramp Length & Diagnostic Position & {$[-2 \sigma,+2 \sigma]$} & All Ny & Pump Depl (\%) & Leakage (\%) \\
\hline $1 Z_{R}(141 \mu \mathrm{m})$ & Top Ramp & 1.3915147 & 1.4495887 & 0.0162 & -4.0056 \\
$1 Z_{R}$ & After 4 $Z_{R}(750 \mu \mathrm{m})$ & 1.3752697 & 1.4425533 & 0.5015 & -4.6408 \\
$1 Z_{R}$ & After $8 Z_{R}(1300 \mu \mathrm{m})$ & 1.3590221 & 1.4400244 & 0.6759 & -5.587 \\
$1.5 Z_{R}(211 \mu \mathrm{m})$ & Top Ramp & 1.3949326 & 1.448308 & 0.1046 & -3.6815 \\
$1.5 Z_{R}$ & After 4.4 $Z_{R}(875 \mu \mathrm{m})$ & 1.3653968 & 1.4449765 & 0.3343 & -5.4889 \\
$1.5 Z_{R}$ & After 7.6 $Z_{R}(1323 \mu \mathrm{m})$ & 1.3591872 & 1.4403573 & 0.6529 & -5.5986 \\
$2 Z_{R}(282 \mu \mathrm{m})$ & Top Ramp & 1.3650521 & 1.446322 & 0.2413 & -5.6057 \\
$2 Z_{R}$ & After 5 $Z_{R}(1058 \mu \mathrm{m})$ & 1.375405 & 1.4348816 & 1.0306 & -4.1023 \\
$2 Z_{R}$ & After 7.6 $Z_{R}(1384 \mu \mathrm{m})$ & 1.3474418 & 1.4274335 & 1.5444 & -5.5173 \\
\hline \hline
\end{tabular}

Table 2: Power Spectral Diagnostic for varying ramp lengths and improved matching

In our study, two important energy loss mechanisms will be consider, pump depletion and energy leakage through the walls of the channel. In this section, energy loss as the pulse enters into the channel and after many Rayleigh lengths of propagation is calculated using power spectral diagnostics [3]. The algorithm applies Fast Fourier Transforms (FFTs) in both space and time on components of the total electric field in the simulation box and then calculated the power spectral density (PSD) to determine existing wave signatures in the electric field. Pump depletion is calculated as $P_{p d}=\left(P S D_{v a c}-P S D_{N y}\right) / P S D_{v a c}$. Energy leakage through the channel walls is $P_{l k}=\left(P S D_{N y}-\right.$ $\left.P S D_{\text {chan }}\right) / P S D_{\text {vac }}$

Table 1 summarized the diagnostic when the laser pulse is focused at the entrance of the channel. We notice that longer density ramps produce more energy loss due to both pump depletion and leakage. The results when the laser spot is refocused to minimize spot size oscillations is shown in Table 2. We observed that reducing spot size oscillation does not have a big impact on pump depletion. The biggest gain is on energy leakage where the energy loss was reduced up to three times compared with the previous case.

\section{CONCLUSIONS}

We have investigated the coupling of intense laser pulses into preionized plasma channel by performing 2D PIC sim- ulation using the VORPAL code. The effects of ramp length and laser focus position was studied in connection with energy loss by pump depletion and leakage. We showed that when the laser spot is focused at the channel entrance, the spot size executed big amplitude oscillations as the laser pulse propagates inside the channel, which in turns, aggravates energy loss by leakage. Refocusing the laser spot before the channel entrance (to compensate for self-focusing) can eliminate or greatly reduce spot size oscillations. In this situation, the energy loss by leakage was reduced up to three times, although pump depletion remains almost the same.

\section{REFERENCES}

[1] C. G. R. Geddes, Cs. Toth, J. van Tilborg, E. Esarey, C. B. Schroeder, D. Bruhwiler, C. Nieter, J. R. Cary and W. P. Leemans, "Nature" 431 (2004) p. 538.

[2] C. Nieter, J. R. Cary, “J. Comp. Phys.” 196 (2004), p.448.

[3] D. A. Dimitrov, D. L. Bruhwiler, J. R. Cary, P. Messmer, C. Geddes, W. P. Leemans and E. Esarey, "AIP Conf. Proc.” 737, ed. V. Yakimenko p.469 (2004).

[4] E. Esarey, P. Sprangle, J. Krall and A. Ting, "IEEE Trans. Plasma Sci.” 24 (1996), p. 252.

[5] P. Sprangle, E. Esarey, J. Krall and G. Joyce, "Phys. Rev. Lett.” 69, 2200 (1992).

[6] E. Esarey, P. Sprangle, J. Krall, A. Ting anf G. Joyce, "Phys. Fluids B" 5, 2690 (1993). 